\title{
INTERPRETAR LA OBRA DE EDUARDO RAMÍREZ VILLAMIZAR: CONFIGURACIONES GEOMÉTRICAS BI Y TRIDIMENSIONALES
}

\section{INTERPRETING THE WORK OF EDUARDO RAMIREZ VILLAMIZAR: TWO- AND THREE-DIMENSIONAL GEOMETRIC CONFIGURATIONS}

\author{
Fabián Alfredo Mena Uscategui ${ }^{1}$ \\ Javier Alberto Mariño Díaz ${ }^{2}$ \\ Mawency Vergel Ortega ${ }^{3}$
}

\section{RESUMEN}

La investigación tiene por objetivo Generar un proceso de enseñanza - aprendizaje a partir de la interacción grupal entre docentes y estudiantes de arquitectura de la Universidad de Pamplona para el estudio de los atributos conceptuales, geométricos y tecnológicos de la obra del maestro Eduardo Ramírez Villamizar hacia la realización de reinterpretaciones creativas a través de perspectivas sensibles de autores teóricos contemporáneos. Para ello se realiza un análisis de los atributos sensibles, geométricos y tecnológicos de la obra del maestro Eduardo Ramírez Villamizar en jornadas de socialización 1 Arquitecto, Filiación: Docente investigador Universidad de Pamplona, Director Grupo de investigación Methodos. Orcid: https://orcid.org/0000-0002-2357-7369 correo: fabianalfredomu@ufps.edu.co

2 Docente investigador Universidad Francisco de Paula Santander,

Grupo de investigación Dlab. Orcid: https://orcid.org/00000001-8624-1910 correo: javieralbertomd@ufps.edu.co 3 Investigadora Universidad Francisco de Paula Santander. Grupos de investigación Euler -Graunt correo: mawencyvergel@ufps.edu.co, orcid: https://orcid.org/ 0000-0001-8285-2968 de trabajo, dialogo y autocritica. Resultado muestra que las experimentaciones, permiten potenciar la creatividad e innovación en los jóvenes.

\section{PALABRAS CLAVE}

Abstracción Geométrica, Proporción, descomposición Geométrica, configuración espacial, fenomenología, experiencia sensorial.

\section{ABSTRACT}

The objective of this research is to generate a teaching-learning process based on group interaction between teachers and students of architecture at the University of Pamplona for the study of the conceptual, geometric and technological attributes of the work of the master Eduardo Ramirez Villamizar towards the realization of creative reinterpretations through sensitive perspectives of contemporary theoretical authors. To this end, an analysis of the sensitive, geometric and technological attributes 
of the work of master Eduardo Ramirez Villamizar is carried out in days of socialization of work, dialogue and self-criticism. The result shows that the experimentations allow to promote creativity and innovation in young people.

\section{KEYWORDS}

Geometric abstraction, proportion, geometric decomposition, spatial configuration, phenomenology, sensorial experience.

\section{INTRODUCCIÓN}

"Toda forma pictórica se inicia con un punto que se pone en movimiento...un conjunto de energías cinéticas cambian al punto en línea, la línea en plano y el plano en una dimensión espacial". (Paul Klee).

El semillero 2112 desde el grupo de investigación METHODOS ha sido parte del sumario de caracterización y análisis de los bienes muebles del cementerio central a través de un proceso de registro y digitalización del patrimonio oculto de la ciudad de Cúcuta en el marco de la investigación "Caracterización y análisis de los Bienes Muebles del Cementerio Central de Cúcuta". Conforme a esta idea que involucra patrimonio y la memoria histórica, hemos decidido continuar con los procesos de registro (Carrascal, Prado y Puentes, 2021) y documentación de bienes muebles, en este caso con el estudio de la obra del maestro Eduardo Ramírez Villamizar referente del arte moderno en colombiano con el propósito de realizar un taller creativo. El objetivo en parte es entender su producción y visualizada como instrumento pedagógico (Duarte, Sánchez y Gómez, 2019) para el dialogo y debate sobre variables asociables al diseño por medio de explorar y concatenar temas relacionados a la arquitectura. Se propone aproximar al educando desde un término histórico - simbólico del referente de mediados del siglo XX hasta el abordaje conceptos que preceden la esencia del diseño y la arquitectura contemporánea en una dinámica de entrecruzamientos de ideas y pensamientos con autores de actualidad.

\section{CRITERIOS CONCEPTUALES DE GEOMETRÍA Y COMPOSICIÓN}

Desde la retrospectiva de la obra de Eduardo Ramírez Villamizar se puede observar la atracción en su proceso artístico por la experiencias de los artistas geométricos posteriores de la segunda guerra mundial y por las teorías de su amigo el francés Jean Dewane, quien sustentaba una idea estricta de lo que debía ser la pintura: la composición total debía tener una armonía perfecta, a cada color debía corresponder un valor equivalente y la sumatoria final debía tener una estructura en completo equilibrio.

Ramírez Villamizar se enfrenta a una tela, a la bidimensionalidad, a las tensiones y los equilibrios, a una estructura de campos definidos en verticales y horizontales (Nolasco, Jacome y Afanador, 2019), a través de los cuales se retoma la esencia de la realidad, para allí representarla o reincorporarla como síntesis geométrica. La línea, el tono, el movimiento, el ritmo, hacen parte de un nuevo repertorio como estructura el cual hereda de Torres García, como tercera y última etapa de la pintura de Ramírez, la geometría. 


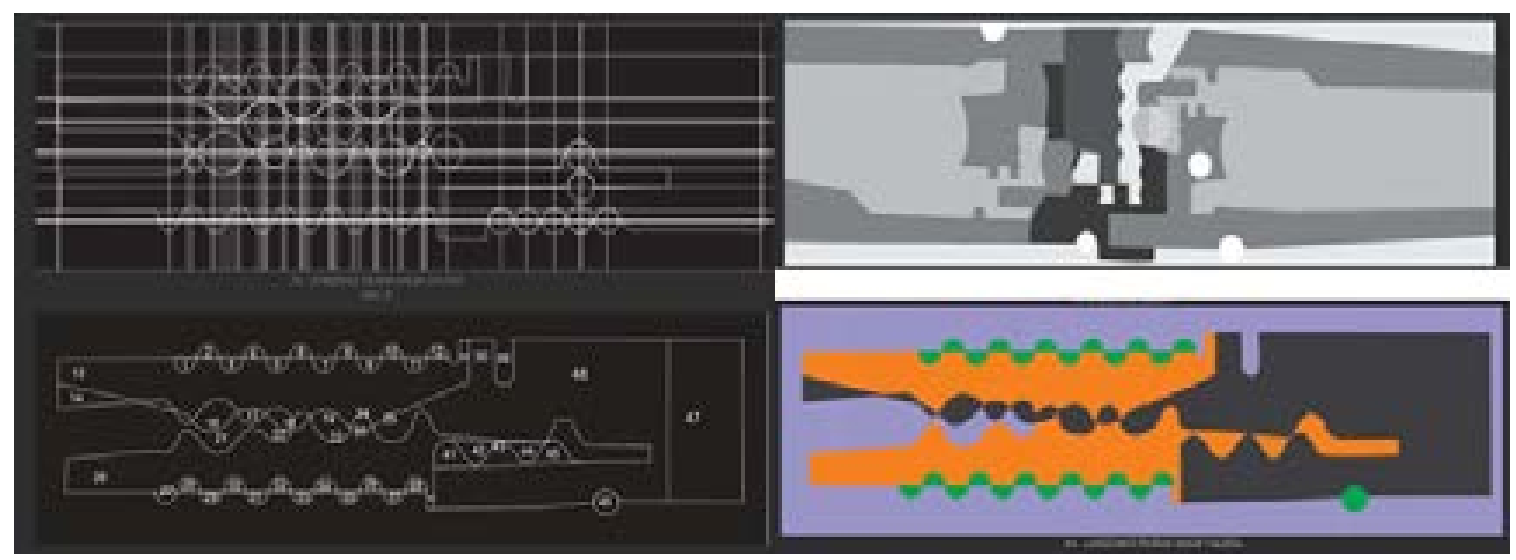

Figura 1: Proceso Bidimensional, Bidimensional geométrica. (Interpretación de la tipología)

\section{METODOLOGÍA}

Lo mismo que en la pintura y el relieve, la conciliación de los elementos escultóricos en el espacio está so-metida a la existencia previa de una tensión. La tensión se plantea, las formas entran en conflicto, se encuentra entre ellas un punto de armonía, las fricciones se disuelven; un ritmo positivo, de vida hallada y ex-presada

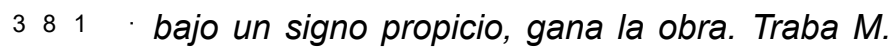
1982. La metodología abarca un proceso tanto cualitativo como cuantitativo (Parra, Rojas y Vergel, 2019), se tienen en cuenta condiciones perceptivas (fenomenológicas y sensoriales) y descriptivas (dimensiones y proporciones) en el desarrollo de los ejercicios.

1. Socialización y marco temático de actividades entre docentes tutores

2. Desarrollo de ejercicios bajo ideas y conceptos desde el aula virtual que se traducen en infografías y materializaciones

3. Realización de exposiciones y sitios web ( blogs )

"La construcción es el arte de configurar un todo con sentido a partir de muchas particularidades. Los edificios son testimonios de la capacidad humana de construir cosas concretas. Para mí, el núcleo propio de toda tarea arquitectónica reside en el acto de construir, pues es aquí, cuando se levantan y se ensamblan los materiales concretos, donde la arquitectura pensada se convierte en parte del mundo real." (Peter Zumthor, Pensar la arquitectura, pag.11 2018). Para los análisis, se realiza un análisis de los atributos sensibles, geométricos y tecnológicos de la obra del maestro Eduardo Ramírez Villamizar en jornadas de socialización de trabajo, dialogo y autocritica, a través de instrumentos consulta y lectura grupal sobre la retrospectiva de la obra del maestro Eduardo Ramírez. Resultado de las experimentaciones sobre las estructuras y hallazgos localizados en las diferentes etapas de la obra conforme al abordaje de conceptos de autores teóricos contemporáneos, de la reversión de la obra a partir del entrecruzamiento de ideas $y$ pensamientos sobre el diseño (Parra y Vergel, 2018) y arquitectura de autores contemporáneos, se desarrollan gráficas atendiendo aspectos de inclusión y diseño (Vergel y Zafra, 2018), se incorporan lenguajes y pictogramas (Vergel, Gómez y Carvalho, 2019) y prototipos resultantes del proceso de enseñanza.

\section{RESULTADOS}

En la obra de Eduardo Ramírez, la juventud identifica categorías emergentes de lenguaje y 
subcategoría expresiones manifiestas a través de diálogo visual, ideas con sensación profunda, poesía, naturaleza, color; categoría movimiento con subcategoría arte, desplazamiento; categoría dimensión con subcategorías forma, espacio, área, volumen, tiempo.

\section{Experimentaciones permiten apreciar} categorías emergentes de réplica (estudiantes que copiaron obra tal cual, en dimensión, forma, color); creatividad (estudiantes que hacen modificaciones a la obra siguiendo parámetros de color y forma semejante) (Figura 2)., innovación, estudiantes quienes a partir de conceptualización e ideas transforman la obra y generan nuevas creaciones siguiendo el estilo de las estructuras (Figura 3 )
Tal como lo expresa Francis D.K. Ching:

"La primera fase de cualquier proceso proyectual es el reconocimiento de un problema y la decisión de solucionarlo. El proyecto es, sobre todo, un acto volitivo, un empeño intencional. En primer lugar, el diseñador debe informarse acerca de la situación actual del problema, definir su contexto y reunir los datos que merezcan ser tenidos en cuenta. Esta es la fase crítica del proceso del proyecto, puesto que la naturaleza de la solución estará inevitablemente condicionada al modo de captar, definir y articular el problema. El destacado poeta y científico danés Piet Hein lo expresó de esta manera: "Arte es resolver problemas que no se pueden formular antes de solucionarlos. La definición del problema forma parte de la respuesta"'. (Francis D.K. Ching. Arquitectura, Forma, Espacio y Orden pag.9, 2018)
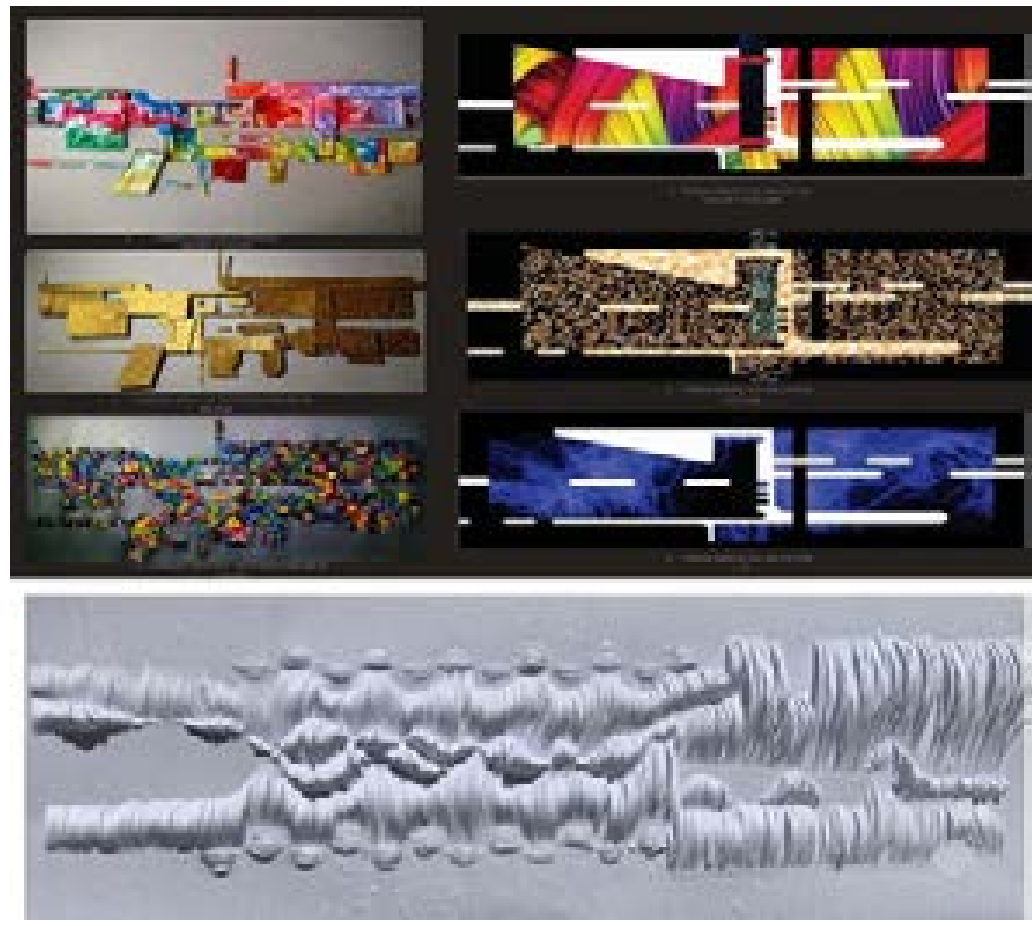

Figura 2: Interpretación matérica. (De lo Bidimensional al relieve) 


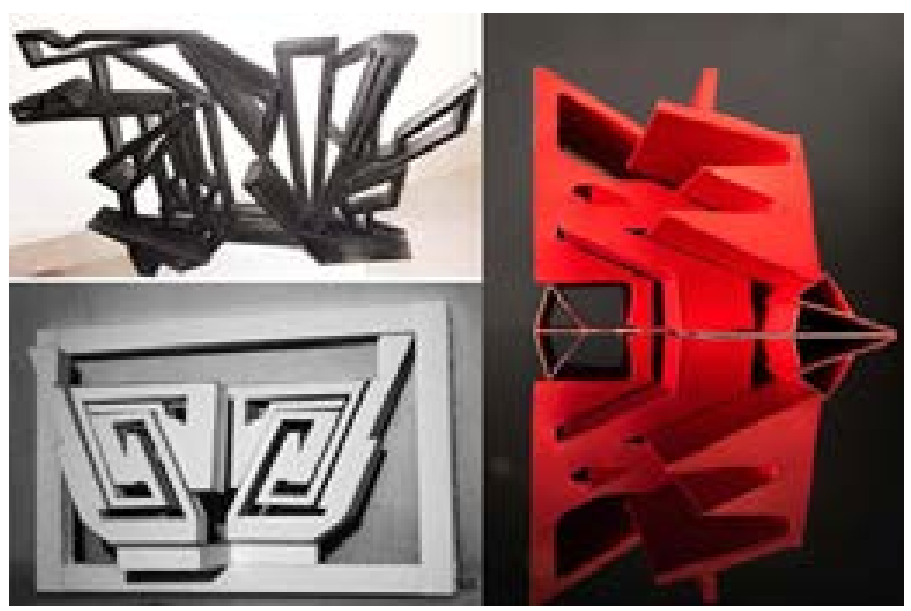

Figura 3: propuestas Tridimensional. (Composición Geométrica espacial)

Obtener una muestra del desarrollo procesado perceptualmente, en donde la metodología correspondeal entendimientológico de reconocer y configurar cada una de las dinámicas físicas y geométricas que se caracterizan de un sector urbano, donde se propone una materialización espacial, particular y compleja.

Se observó que el desarrollo de cada obra en el proceso de aprestamiento de los jóvenes incluye en su orden una metodología propia e individual pero que generalizada conlleva: a partir del cual primero observaron las obras, dieron lectura a textos y documentos referentes a la obra, realizan réplicas de las obras, identifican patrones, características, se apropian de significados y estilos, realizan esbozos de obras en dos dimensiones, realizan obras en tres dimensiones, experimentan cambios, realizan creaciones propias, analizan obras, realizan diálogo crítico a partir de obras de sus compañeros y de la propia, obtienen conclusiones.

\section{CONCLUSIONES}

La interpretación de obras permite a la juventud apropiarse del lenguaje, generar diálogos constructivos a través de expresiones manifiestas, potenciar capacidad de análisis y los sentidos visuales, táctil, ahondarse en la poesía, potenciar la observación de la naturaleza y su transposición didáctica a la realidad de las obras generando arte a través del uso del color, del movimiento, la forma el uso del espacio, volumen y el tiempo.

Experimentaciones a partir del desarrollo de obras permiten potenciar la creatividad e innovación en los jóvenes.

\section{REFERENCIAS BIBLIOGRÁFICAS}

Carrascal Bermúdez, J. A. ., Prado Chinchilla, H. O. ., \& Puentes Velásquez, A. M. (2021). Desarrollo de un sistema semántico interactivo de los aspecto curriculares del Programa de Ingeniería de Sistemas de la UFPSO. Covalente, 1(1). https:// doi.org/10.22463/2711015X.3006

Duarte, Y., Sánchez-Frank . J. and. Gómez, C . 2019. "El trabajo colaborativo en las competencias económicas y financieras en estudiantes del Instituto Agrícola Región del Catatumbo-Colombia", Covalente, vol.1, no. 36, 2019

Ferrater, C., Asociados (OAB). (2006) Sincronizar la geometría. Barcelona, España. Actar 
Francis D.K. Ching. 2018. Arquitectura, Forma, Espacio y Orden, Editorial Gustavo Gili, SA, Barcelona,

Guallart, V. (2009) Geologics, Geografía información arqutiectura. Actar.

1. Holl S. 1994. Cuestiones de percepción, Fenomenología de la arquitectura, Editorial Gustavo Gili, SA, Barcelona

Nolasco-Serna, C. Jácome-Castilla, N. J. Afanador-Garcia, N. 2019. Aplicación de la ecuación de Poisson a la transferencia de calor en cristales líquidos., Covalente, vol. 01, no. 1, 6-11, 2019.

2. Pallasmaa J. 2010. Los ojos de la piel. la arquitectura de los sentidos, BarceIona: Editorial Gustavo Gili, SA,

Parra-López, H., Rojas-Suárez J.,P. y VergelOrtega, M. 2019 J. Phys.: Conf. Ser. 1329012013

Parra H and Vergel M 2018 Hacia un modelo para evaluar la pertinencia social en la oferta académica de la universidad Francisco de Paula Santander 1 (Alemania: Editorial Académica Española) 234

Traba M. 2016 Historia abierta del arte colombiano, biblioteca básica de cultura colombiana,

Zumthor P. 2004. Pensar en la Arquitectura, Editorial Gustavo Gili, SA, Barcelona,

Vergel M and Zafra S 2018 Inclúyeme en la U (Bogotá: Editorial Ibañez) 133

Vergel-Ortega, M., Gómez-Vergel, y C.S. Caravalho-Casanova, J.F. 2020. Storydoing in the emotions of young university students during the process of learning calculus in engineering. Respuestas, 25(1), pp. 89-95 